\title{
MULTI-AGENT PARADIGM FOR COGNITIVE PARAMETER BASED FEATURE SIMILARITY FOR SOCIAL INFLUENCE
}

\author{
Swati Basak ${ }^{1}$, Bireshwar Dass Mazumdar ${ }^{2}$, Chandra Bhan Patheriya ${ }^{3}$ \\ ${ }^{1,3}$ Dept. of Computer Engineering, SITM, Lucknow India, ${ }^{2}$ SMS Varanasi India \\ swati.basak.bhu@gmail.com,bireshwardm@gmail.com,chandrabhan2006@gmail.com
}

\begin{abstract}
The ABM methodology is a favorable approach to model and analyze complex social phenomena that may involve non-linear feedback loops. It has been applied successfully to model a number of social phenomena involving different social processes and organizational structures. Availability of cheap computing power and rich software resources has made ABM a widely used and hence more popular methodology. A modeler using ABM however have be careful about choosing the right amount of detail (less and more both can be problematic) and validating (internal and external) the model. Interpreting and analyzing results is also an involved task. In this paper, we have demonstrated how ABM can be applied to model and analyze the voting preference formation and resultant voting decisions of individuals in a population. The model assumes a two party system. We designed three versions of the simulation and observed the results for a large number of runs with different parameter variations. The results obtained present interesting picture and resultant inferences.
\end{abstract}

\section{INTRODUCTION}

The development of social simulation over the past half century can be grouped into three broad periods: macro simulation, micro simulation and agent-based models. Sociologists, particularly computational sociologists, first tried macro simulations to model problems in supply-chain management, inventory control in a warehouse, urban traffic, and spread of epidemics, migration and demographic patterns. Macro simulations consist of a set of differential equations that take a holistic view of the system. However, taking the complete system as the unit of analysis had inherent limitations. Microsimulations focused on the use of individuals as the unit of analysis but continued with macrolevel forecasting used in macrosimulations. Though microsimulations modeled changes to each element of the population but they did not permit individuals to directly interact or to adapt. The main focus remained forecasting macro effects of public policies that alter individual behaviors. $\mathrm{ABM}$, the third methodology, takes a pure bottom-up approach and keeps the individual at the centre. It allows modeling individual actors in the population and their interactions with each other as well as with the environment. This makes it a highly suitable technique for analysis of emergent group behaviors resulting only from local interactions of individuals. Besides, there are many other advantages of this approach.

In Agent-based modeling (ABM) a system is modeled as a set of autonomous agents, who can perceive the environment and act on the basis of some behavioral rules. The agents represent the actors in the system; environment represents the surroundings including neighbouring agents; and the behaviour rules model the interaction of the agent with other agents as well as with the environment. ABM can be used to model a number of phenomena in varied domains like Markets \& Economy, Organizations, World Wide Web and Social Systems etc. Availability of fast and cheap computing power, coupled with other advances in computational sciences has paved the way for use of $\mathrm{ABM}$ as a preferred modeling and simulation technique. Since last few years ABM has become the frontrunner tool of the Sociologists and Psychologists who try to model social cognitive parameter based behaviours, particularly the behaviour of groups and societies. A large number of researches are now being carried out using this generative approach to model and analyze social phenomenon, such as spread of rumors, diffusion of ideas, emergence of cooperation, emergence of Conventions \& social norms, evolution and dynamics of spread of religions and cultures etc.

This paper presents our experimental work on using ABM for understanding and analyzing cognitive parameter based voting preferences of individuals. We have modeled the process and factors shaping and affecting individual voting preferences for a particular political party according to cognitive parameter based. To keep the model simple and consequently analysis more accurate, we have taken individual voting preferences as ' 0 ' and ' 1 ' representing a two party political system. Every individual's final voting decision is shaped and affected by a number of factors, such as his own perception about parties, his family views \& beliefs, perception of his friends, and his neighbourhood preferences. Our simulation models the role and relative weights of these factors in shaping an individual's voting preferences. We have also incorporated social influence theory in our model which states that similar persons are more 
likely to interact, and as a result of frequent interactions they are likely to become more similar.

\section{ROLE OF AGENT BASED MODELING IN SOCIAL INFLUENCE}

Traditional modeling approaches to social influence systems relied on equation-based models that operate with a macro perspective. They operate on population attributes \& their distributions and lack the focus on individual's role. Equationbased models, though useful for macro-scale behaviour predictions, fail to model social influence systems (or processes) that lack central coordination, systems that are very complex in terms of their interdependencies and systems which produce novel emergent behaviours in absence of a clear understanding of the collective phenomenon. Axtell [1] takes this argument a level further and suggests that there are three distinct uses of Agent-based computational models of social influence systems: (a) when numerical realizations can be proposed and solved: agent models can be used as social simulations; (b) when a mathematical model can be formulated but can only be partially solved: agent based models can be a useful tool of analysis; and (c) when mathematical models are either apparently intractable or provably insoluble: agent based modeling is perhaps the only technique available for systematic analysis. Availability of fast \& cheap computing power along with rich easy-to-use software environments also favours the use of ABM in Social Sciences.

Although technically simple, ABM is conceptually deep. Modeling a complex social process requires high conceptual clarity and analytical ability. A key issue, therefore, is to decide when to use $\mathrm{ABM}$ for modeling social influence systems. An indicative list of situations when it is better to think and model in terms of agents is: (a) when there is a natural representation of actors as agents; (b) when the interactions between the agents are complex, non-linear, discontinuous, or discrete; (c) when agents exhibit complex adaptive cognitive parameter based behaviours; (d) when the population or topology of interactions is heterogeneous; and (e) when agents have spatial component to their cognitive parameter based behaviours \& interactions. ABMs in social sciences involve human agents, whose behaviours are often complex, irrational and subjective. Therefore, one needs to think carefully about the social phenomenon at hand before going for ABM. Further, the model needs to be built at the right level of description, with only the right amount of details. Unnecessarily adding complexity to a model can make the analysis difficult and the model useless.

$\mathrm{ABM}$ is currently being applied to model a variety of complex social phenomenon where simple local interactions generate emergent system-level behaviours. Some representative \& relevant work can be found in [1], [2], [3] \& [4]. Macy \& Willer [3] group most of the work on Agent-based modeling of collective behaviours in two categories: (a) models of emergent structure which includes works on cultural differentiation, homophilous clustering, idea diffusion, convergent behaviours and norms; and (b) models of emergent social order which include viability of trust, cooperation and collective action in absence of global control. Goldstone \& Janssen [10] also identify three similar themes for Agentbased computational models of collective behaviour namely: (a) patterns and organizations which include settlement patterns \& segregation, human group behaviours and traffic patterns; (b) social contagion which include spread of ideas, fashions, cultures \& religions; and (c) cooperation which include evolution of cooperation, trust \& reputations and social norms \& conventions.

\section{SOCIAL INFLUENCE OF COGNITIVE PARAMETERS}

The Social influence assumes that individuals (or agents) often imitate cognitive parameter based good behaviours and cultures through their interactions with other individuals by using coordination and cooperation (cognitive parameters). Its dynamics depends on familiarity of the interacting individuals, density of the neighbourhood, and popularity and spatial proximity of the other individuals. The model of social influence developed in two ways: (a) it explicitly takes into account that the effect of one cultural feature depends on the presence or absence of other cultural features and behaviours; and (b) it takes into account that similar individuals are more likely to influence each other than dissimilar individuals. Interesting models of social influence have been proposed by [4], [6], [7], [8], [9] etc. Axelrod [6] and [9], in his social influence model of dissemination of culture [6], [9] focused on factors of local influence (tendency for people who interact to become more similar) and homophile (tendency to interact more frequently with similar agents). The more agents interact, the more similar they become; and the more similar they become, the more likely they are to interact. Axelrod expected convergence and homogeneity as the outcome but simulation shown that despite the strong converging pressure, stable regions of diversity persisted.

Axelrod [6] and [9] basic model included sites arrayed on a grid. These sites are the basic actors of the model. Each site can interact only with its immediate neighbours. Agents who are similar to each other interact with each other and become more similar. Axelrod's model captures culture as a group of features with multiple trait values per features (cognitive parameters). However, the emphasis is not on the content of a specific culture but on the way in which culture is likely to emerge and spread. The simulations with varying parameters regarding grid size, number of features and number of traits per feature resulted in polarization, despite the only mechanism for change being convergence towards a neighbour. We have used the basic idea of Axelrod's culture model to represent friends of an individual [7]. An 
individual's friend is believed to have cognitive parameter based behavioural feature set (cognitive parameter set) similar to the individual. The feature set similarity represents their like mindedness of the individuals on various issues and hence possibility of having similar voting preferences (example of model) or at least being affected by each other's preferences. An individual while deciding about his vote (example model) is thus likely to be affected by his interactions with friends.

Take an example of cognitive social influence model of voting; an individual makes up his voting preference through a complex process involving non-linear feedback loop of interactions with a number of entities. Individuals interact with frequently with their family members and relatively less frequently with other persons belonging to their social and religious groups. An individual's final voting value is no doubt a function of these cognitive factors. However, what is more important to understand is that what is the extent of this effect and the by what underlying process an individual finally makes up his voting preference. This model can be solved by Greedy Approach and Case Base Reasoning (CBR) method, some weighted values are assigned to the cognitive parameters to decide the final voting preference of an individual; the CBR method will be discussed in next section.

\section{VOTING MODEL WITH FEATURE SIMILARITY}

In this Voting Model initially setup is design in such a manner that all the individuals in grid or a particular area are assigning with randomly votes and randomly assign feature set which will decide final vote of individuals after dynamic interaction among neighbour agents.

\section{Model Setup}

A population of agents is created on a wrap-around agent grid of size $40 \mathrm{X} 40$. The key entities in the model are represented by patches; each patch of the grid represents an agent. The patches represent stationary landscape on the grid. In this version agent hold a set of features. Each feature is a variable that can adopt a binary value (1 or 0$)$. Initially, agents adopt randomly chosen a set of features. If feature size is $n$ than there will be $2 \mathrm{n}$ different type of feature set. Every agent has one of this $2 \mathrm{n}$ feature set.

\section{Model Parameters}

In grid, we have placed agents at each coordinate. Every individual has certain attributes that characterize it. Most interestingly, it has a cognitive parameter based behavioural feature set of size "n", which can be a 1 to 10 bit string. Every value in the string is a binary value ( 0 or 1$)$ and represents a particular feature. The feature set values of two agents is used to decide whether they are sufficiently likeminded or not. Two individuals having will be similar if their feature set string contains a minimum number of similar feature values (greater than a similarity threshold). An individual is more likely to be influenced by those who are similar to him. In addition to the feature set, every agent also has a voting preference value (1 or 0 ), initialized randomly. Before deciding his final voting preference, an agent interacts with various agents in its neighbourhood. According to social influence theory, it is more likely to interact and be affected by the agents similar to it. Interactions between similar agents make them more similar. However, rather than changing the feature set values of agents, we have changed their voting preference values so that similar agents are more likely to have similar voting preferences. In fact, the model picks up an agent and samples its neighbourhood. Suppose $\mathrm{n}$ out of total $\mathrm{m}$ agents in the neighbourhood are similar to the agent. Then the voting preference of the agent changes to the majority preference of the similar agents. In case of tie the agent retains its voting preference. We obtained multiple runs of the model by varying (i) the feature size, (ii) feature threshold size and (iii) initial voting preference value distribution.

\section{RESULTS}

In these model multiples Runs are taken by varying feature size, threshold size and initial voting preference value distribution. The feature size " $\mathrm{n}$ " has been varied from 1 to 10 with feature similarity threshold being varied from 1 to $\mathrm{n}$. Fig. 1 to Fig. 7 presents some initial and final snapshots of voting model with feature similarity.

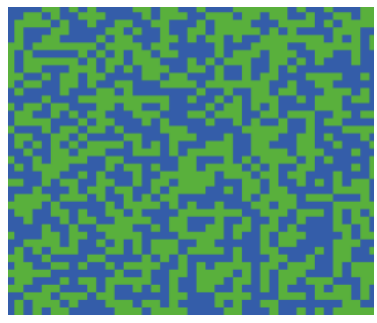

1(a)

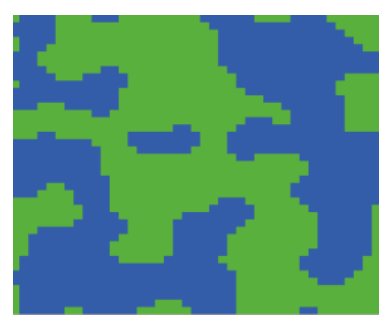

1(b)
Fig. 1: A snapshot of the run of feature set based simulation. The feature set size is 7 and similarity threshold is 1 . The initial values of green $=840$ and blue $=841$ (Fig. 1(a)), transform to final values of green $=856$ and blue $=825$ and stability time tick $=10$ (Fig. 1(b))

Table 1 shows few more of sampling runs by taking the above data which describes the stability of model; time to stability increases with decreasing the threshold size. It also shows if threshold size nearer to feature size there will be less polarization 
Table 1: Sample Runs of Feature Based Voting Model

\begin{tabular}{|c|c|c|c|c|c|c|c|}
\hline \multirow[t]{2}{*}{$\begin{array}{l}\text { No. of } \\
\text { Runs }\end{array}$} & \multirow{2}{*}{$\begin{array}{c}\mathbf{F e} \\
\text { at } \\
\text { ur } \\
\text { e } \\
\text { Si } \\
\text { ze }\end{array}$} & \multirow{2}{*}{$\begin{array}{c}\text { Th } \\
\text { res } \\
\text { hol } \\
\text { d } \\
\text { Siz } \\
\text { e }\end{array}$} & \multicolumn{2}{|c|}{$\begin{array}{l}\text { No. of Green } \\
\text { Vote }\end{array}$} & \multicolumn{2}{|c|}{$\begin{array}{c}\text { No. of } \\
\text { Blue Vote }\end{array}$} & \multirow{2}{*}{$\begin{array}{l}\text { Tim } \\
\text { e to } \\
\text { Sta } \\
\text { ble }\end{array}$} \\
\hline & & & $\begin{array}{c}\text { Initi } \\
\text { al }\end{array}$ & Final & $\begin{array}{c}\text { Initi } \\
\text { al }\end{array}$ & $\begin{array}{c}\text { Fin } \\
\text { al }\end{array}$ & \\
\hline Run-1 & 7 & 1 & 840 & 856 & 841 & 825 & 10 \\
\hline Run-2 & 7 & 2 & 840 & 960 & 841 & 721 & 7 \\
\hline Run-3 & 7 & 3 & 840 & 860 & 841 & 821 & 7 \\
\hline Run-4 & 7 & 4 & 840 & 834 & 841 & 847 & 3 \\
\hline Run-5 & 7 & 5 & 840 & 838 & 841 & 843 & 2 \\
\hline Run-6 & 7 & 6 & 840 & 841 & 841 & 840 & 2 \\
\hline Run-7 & 7 & 7 & 840 & 840 & 841 & 841 & 1 \\
\hline Run-8 & 5 & 3 & 169 & 104 & $\begin{array}{c}151 \\
2\end{array}$ & $\begin{array}{c}157 \\
7\end{array}$ & 4 \\
\hline Run-9 & 5 & 3 & 505 & 417 & $\begin{array}{c}117 \\
6\end{array}$ & $\begin{array}{c}126 \\
4\end{array}$ & 3 \\
\hline $\begin{array}{c}\text { Run- } \\
10\end{array}$ & 5 & 3 & 673 & 610 & $\begin{array}{c}100 \\
8\end{array}$ & $\begin{array}{c}107 \\
1\end{array}$ & 4 \\
\hline $\begin{array}{c}\text { Run- } \\
11\end{array}$ & 3 & 2 & 169 & 144 & $\begin{array}{c}151 \\
2\end{array}$ & $\begin{array}{c}153 \\
7\end{array}$ & 3 \\
\hline $\begin{array}{c}\text { Run- } \\
12\end{array}$ & 3 & 2 & 505 & 458 & $\begin{array}{c}117 \\
6\end{array}$ & $\begin{array}{c}122 \\
3\end{array}$ & 2 \\
\hline
\end{tabular}

\section{CONCLUSIONS}

The ABM methodology is a favorable approach to model and analyze complex social phenomena that may involve nonlinear feedback loops. It has been applied successfully to model a number of social phenomena involving different social processes and organizational structures. Availability of cheap computing power and rich software resources has made $\mathrm{ABM}$ a widely used and hence more popular methodology. A modeler using ABM however have be careful about choosing the right amount of detail (less and more both can be problematic) and validating (internal and external) the model. Interpreting and analyzing results is also an involved task. In this paper, we have demonstrated how ABM can be applied to model and analyze the voting preference formation and resultant voting decisions of individuals in a population. The model assumes a two party system. We designed three versions of the simulation and observed the results for a large number of runs with different parameter variations. The results obtained present interesting picture and resultant inferences. The initial voting preference distribution in the population, role of friends (likeminded individuals), affect of family members and neighbours; all produce interesting results to analyze. Social influence theory incorporated into voting dynamics plays an important role. Individuals' voting decisions are affected more by those who are similar to them. However, if the similarity threshold is relatively lower, the model results in local voting preference polarizations. When the model is based only on majority, the initial distribution of voting preferences becomes the key deciding factor. Both these results taken together present similarity to the actual voting patterns observed in closely knit societies and segregations such as far off villages and tribal groups. The role of implicit favorable waves introduced by mass media can also be understood by the results of the second version of the simulation. When the weights of factors playing role in shaping an individual's voting preference are varied, the results demonstrate that high weight to individual vote is able to sustain variations in voting preferences. When the ownweight is reduced to give more favour on the basis of cognitive values to the family and neighbours, polarizing tendency gains comes in and slight amount of local polarization is seen in even in case of almost uniform initial voting preference distribution. Voting behaviour and patterns of relatively informed and well-educated persons conform to this trend. The experimental simulations attempt to model an interesting social cognitive phenomenon and present interesting results that need to be further analyzed to draw relevant inferences, analogies and explanations.

\section{REFERENCES}

[1]. J.M. Epstein, "Generative Social Sciences: Studies in Agent-based Computational Modeling", Princeton University Press, (2007).

[2] J.M. Epstein \& R. Axtell, "Growing Artificial Societies: Socail science from bottom-up", Cambridge, MA: MIT Press, (1996).

[3]. M.W. Macy \& R. Willer, "From factors to actors: computational sociology and agent based modeling", Annual Review of Sociology, Vol. 28, pp. 143-166, (2002).

[4]. N. Gilbert \& K. Troitzsch, "Simulation for the Social Scientist", Buckingham, U.K.: Open Univ. Press, (1999).

[5].R. Axelrod, "The Complexity of cooperation", Princeton N.J., Princeton University Press, (1997). 
[6]. R. Axelrod, "The Dissemination of Culture: A model with local convergence and global polarization", Journal of Conflict Resolution, Vol. 41, pp.203-226, (1997).

[7]. R. Axelrod \& L. Tesfatison, "A guide for newcomers to agent based modeling in the social sciences", In the Handbook of Computational Economics Vol. 2: Agent Based Computational Economics, (2005).

[8].R. Axtell, "Why agents? On the varied motivations for agent computing in the social sciences, working paper 17", Centre on Social and Economic Dynamics, Brookings Institution, Washington D.C., (2000).

[9]. R. Axtell, R. Axelrod, J.M. Epstein \& M.D. Cohen, "Aligning simulation models: a case study and results", Computational and Mathematical Organization Theory, Vol.1, No.2, pp.123-41, (1996).

[10]. R.L. Goldstone \& M.A. Janssen, "Computational models of collective behaviour", Trends in Cognitive Sciences, Vol.9, No.9, (2005). 